\title{
ARTíCULO \\ Variabilidad espacial termohalina, masas de agua y circulación geostrófica en Bahía de La Paz, Golfo de California
}

\author{
Spatial thermohaline variability, water masses and geostrophic circulation \\ in Bahia de La Paz, Gulf of California
Maclovio Obeso-Nieblas ${ }^{1}$, Juan Heberto Gaviño-Rodríguez² Hipolyto Obeso-Huerta ${ }^{3}$ y Sandra Isaura Muñoz-Casillas ${ }^{1}$

\begin{abstract}
${ }^{1}$ Centro Interdisciplinario de Ciencias Marinas, Instituto Politécnico Nacional, Ave. Inst. Politécnico Nacional S/N, Col. Playa Palo de Santa Rita, La Paz B.C.S. 23090, México. mniebla@ipn.mx

${ }^{2}$ Instituto Oceanográfico de la Universidad de Colima, Kilómetro 20, Carretera Manzanillo, Cihuatlán, Manzanillo Colima 28860, México

${ }^{3}$ Instituto Tecnológico de La Paz, Boulevard Forjadores de Baja California Sur No.4720, La Paz, B.C.S. 23080, México

Abstract.- CTD measurements were processed to analyze the thermohaline variability, presence of water masses and geostrophic circulation during a period of neutral ENSO. The abrupt stratification and the steep thermocline, halocline and pycnocline in May and the thermocline and pycnocline in July, were generated by the entry of water of the gulf, originating from coastal upwelling, due to southerly winds. In October, the salinity decrease with a minimum and a maximum subsurface, was caused by water intrusion from the gulf, as the bay is considered an evaporation basin without river discharge with evaporation of $176.16 \mathrm{~mm}$ and null precipitation in this month. The mixed layer and stratification decreased in February, was the result of homogenization and cooling water by winds from the north and the lowest solar radiation received. The water exchange with the gulf, on May, July and February, involved Gulf of California Water on the surface and Subtropical Subsurface Water in the deep; exceptionally in October, the Tropical Surface Water, replaced the Gulf of California Water. The bathymetric North Mouth's sill prevented the flow incursion of Pacific Intermediate Water into the bay. In May, July and October, the geostrophic circulation in the bay, was dominated by a cyclonic eddy, which facilitated the exchange of water with the gulf, while in February the circulation was anticyclonic.
\end{abstract}

Key words: Hydrography, water masses, thermocline, stratification, upwelling

\begin{abstract}
Resumen.- Se procesaron mediciones de CTD para analizar la variabilidad termohalina, presencia de masas de agua y circulación geostrófica durante un período de ENSO neutral. La estratificación y la abrupta termoclina, haloclina y picnoclina en mayo y la escarpada termoclina y picnoclina en julio, fueron generadas por la entrada de agua del golfo, originada en la surgencia costera, producto de los vientos del sur. En octubre, la disminución de la salinidad con un mínimo y un máximo subsuperficial, fue causada por la intrusión de agua del golfo, puesto que la bahía es considerada una cuenca de evaporación, sin descargas fluviales, con evaporación de 176,16 mm y precipitación nula en este mes. La capa de mezcla y la disminución de la estratificación en febrero, fue resultado de la homogenización y el enfriamiento del agua por los vientos del norte y la menor radiación solar recibida. El intercambio de agua con el golfo, en mayo, julio y febrero, involucró Agua del Golfo de California en la parte superficial y Agua Subsuperficial Subtropical en la parte profunda; de manera excepcional en octubre, el Agua Superficial Tropical, sustituyó al Agua del Golfo de California. El umbral batimétrico de la Boca Norte, impidió la incursión del Agua Intermedia del Pacífico a la bahía. La presencia de un remolino ciclónico en la bahía durante mayo, julio y octubre dominó la circulación geostrófica, lo que facilitó el intercambio del agua con el golfo, mientras que en febrero la circulación fue anticiclónica.
\end{abstract}

Palabras clave: Hidrografía, masas de agua, termoclina, estratificación, surgencia

\section{INTRODUCCIÓN}

En los océanos y bahías se ha establecido que la variabilidad espacio-temporal de la productividad primaria depende en gran medida de las condiciones ambientales a las cuales están sujetas las poblaciones fitoplanctónicas (Millán-Núñez et al. 1993, Bustillos-Guzmán 1995 y
Falkowski \& Raven 1997). Entretanto los cambios en las concentraciones y la distribución de la clorofila en Bahía de La Paz (BLP), tanto espacial como temporal están afectados por las condiciones ambientales prevalecientes (Hinojosa-Larios 2007). 
Por otra parte, debido a que las primeras etapas de vida de los peces son típicamente planctónicas, las relaciones entre la distribución de las larvas de peces y los huevos con los procesos físicos de mesoescala se han analizado en diferentes regiones del mundo (Fiedler 1986, Govoni 1993 y Somarakis et al. 2002). En BLP el desarrollo anual de las asociaciones de larvas de peces, muestra una correlación temporal y espacial con la dinámica geostrófica superficial y los datos hidrográficos (Sánchez-Velasco et al. 2006).

La BLP, parte integrante del Golfo de California (GC), es el cuerpo de agua costero más grande de Baja California Sur, cuenta con una importante complejidad oceanográfica, una enorme actividad biológica y pesquera; con una considerable productividad primaria, así como, con una creciente actividad turística, es por esto, una región de gran interés científico y económico para el noroeste de México (Obeso-Nieblas 2003). Es la bahía más importante del GC debido a su tamaño, profundidad $(450 \mathrm{~m})$ y el intercambio de sus aguas. Una barrera natural formada por tres islas grandes y protuberantes, Isla Espíritu Santo, Isla La Partida e Isla San José, hace de la bahía un área de refugio elemental para muchas especies marinas que tienen procesos biológicos y físicos de interés. La interacción con el golfo es continua y muy dinámica, ocurre a través de 3 entradas (Obeso-Nieblas et al. 2004).

En BLP, durante los meses de noviembre a mayo, los vientos soplan por la mañana del noroeste y después del crepúsculo cambian a vientos del sur; durante el resto del año los vientos del sureste y del suroeste son los dominantes (Obeso-Nieblas \& Jiménez-Illescas 1989). Las calmas son frecuentes durante primavera y verano (Obeso-Nieblas 2003). Existen vientos que pueden alcanzar los $10 \mathrm{~m} \mathrm{~s}^{-1}$ (Jiménez-Illescas et al. 1997). El campo de viento se determina principalmente por el desplazamiento atmosférico del centro de alta presión del Pacífico y centro de baja presión del Desierto de Sonora (Roden 1972). En el GC, la intensidad del campo de viento es máxima durante el invierno $\left(8-12 \mathrm{~m} \mathrm{~s}^{-1}\right)$ cuando predominan los vientos del noroeste. En los veranos, los vientos del sureste $\left(5 \mathrm{~m} \mathrm{~s}^{-1}\right)$ predominan (Douglas et al. 1993).

En trabajos previos hidrográficos de BLP se reportan aguas cálidas, salinas y estratificadas con características del Agua del Golfo de California (AGC) y Agua Subsuperficial Subtropical (ASS) con un intercambio dinámico con el GC (Jiménez-Illescas et al. 1994, Jiménez-
Illescas 1996, Jiménez-Illescas et al. 1997, Monreal-Gómez et al. 2001, Salinas-González et al. 2003, Obeso-Nieblas 2003, Obeso-Nieblas et al. 2004, 2008; Guevara-Guillén 2011).

Por lo anterior, si existe variabilidad espacial en temperatura, densidad, salinidad, estratificación y circulación baroclínica en BLP durante un período de ENSO neutral, así como la presencia de diferentes masas de agua en la región, con diferencia entre la provincia profunda y la provincia somera, por tanto el objetivo del presente estudio fue determinar la variabilidad espacial termohalina, las masas de agua y la circulación geostrófica en BLP, fundamental para el manejo adecuado de actividades antropogénicas, así como para el aprovechamiento sustentable de sus recursos.

\section{MATERIALES Y MÉTODOS}

\section{Área DE ESTUdio}

$\operatorname{La} \operatorname{BLP}\left(24,1-24,8^{\circ} \mathrm{N}, 110,2-110,8^{\circ} \mathrm{W}\right)$ se ubica en la costa este de la Península de Baja California a $200 \mathrm{~km}$ de la boca del GC (Fig. 1). Tiene una dimensión aproximada de $80 \mathrm{~km}$ de largo y su parte más ancha es de $35 \mathrm{~km}$. La comunicación con el GC ocurre a través de 3 entradas: la Boca Norte, una comunicación ancha y profunda que alcanza profundidades de $350 \mathrm{~m}$, Canal San José estrecho y poco profundo en el extremo sur $(62 \mathrm{~m})$ y el Canal San Lorenzo (CSL) muy dinámico y somero con una profundidad promedio de $10 \mathrm{~m}$ (Obeso-Nieblas et al. 2004). La región presenta un clima del tipo BW (desértico) (García 1973). La temperatura promedio anual es de $23,80^{\circ} \mathrm{C}$, la temperatura mínima promedio en invierno es de $8,00^{\circ} \mathrm{C}$ y la temperatura máxima para verano es de $37,00^{\circ} \mathrm{C}$ (INEGI 1994). El régimen de marea en la bahía es predominantemente semidiurno (Obeso-Nieblas 2003).

La evaporación promedio anual en BLP, es de $215 \mathrm{~mm}$ y la humedad relativa está entre 62 y 70\% (Obeso-Nieblas \& Jiménez-Illescas 1989). El fenómeno de 'brisa' es importante en la región y es producto de la diferencia de disipación de la energía en la frontera mar-tierra (ObesoNieblas et al. 2002). Las lluvias son escasas todo el año en BLP, principalmente en invierno y primavera. La máxima precipitación se presenta entre julio y octubre, asociada a vientos del sureste, tormentas tropicales y huracanes. La precipitación promedio anual es de $180 \mathrm{~mm}$ (ObesoNieblas 2003). La Ciudad de La Paz, está situada al sureste de la bahía. 


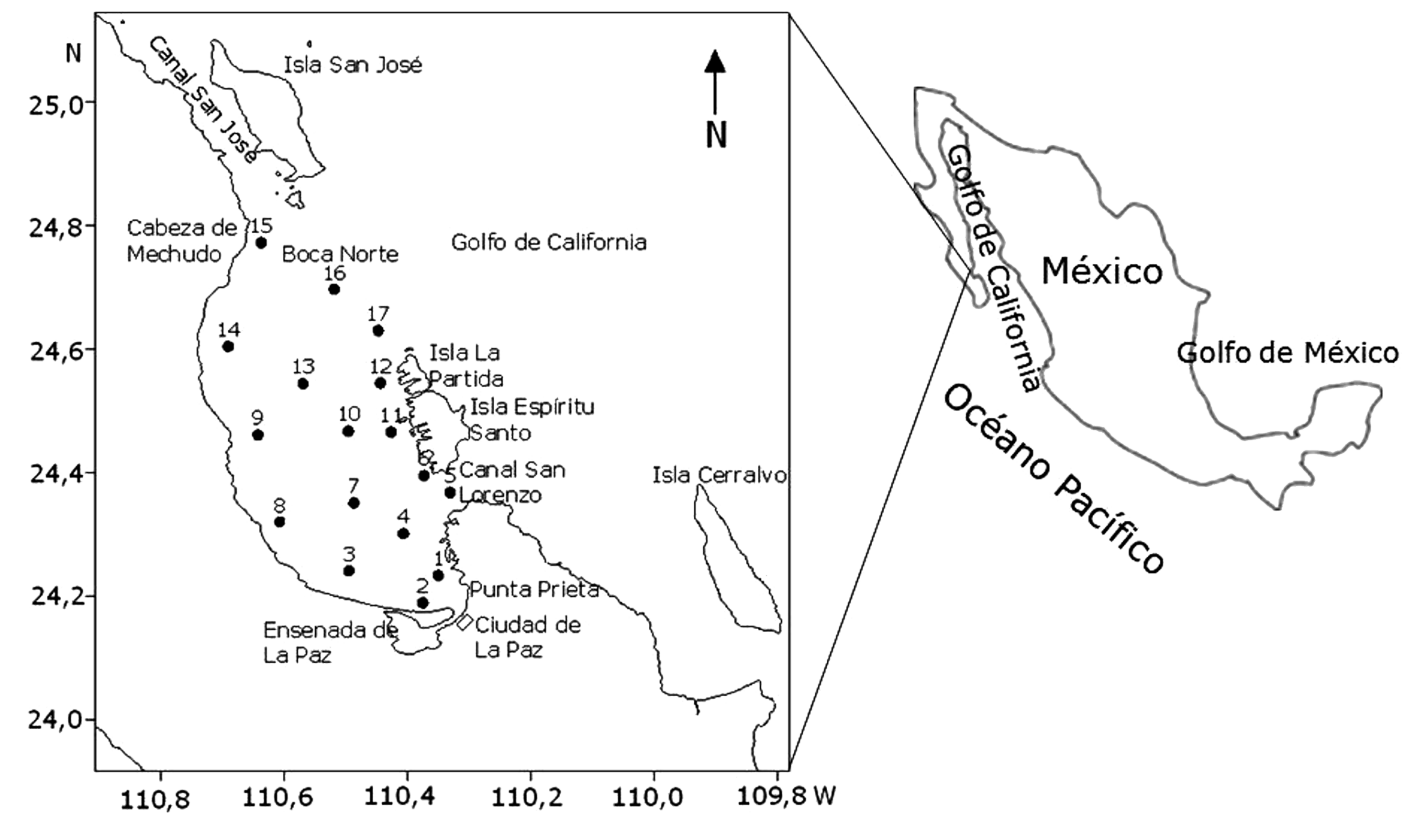

Figura 1. Localización de Bahía de La Paz, México y distribución de las estaciones oceanográficas / Bahia de La Paz, México location and hydrographic station distribution

\section{Trabajo de Campo}

Los datos hidrográficos fueron obtenidos con un CTD marca Seabird modelo Seacat 19, durante 4 cruceros realizados del 18 al 20 de mayo, del 20 al 22 de julio y del 27 al 29 de octubre de 2001, así como, del 10 al 13 de febrero de 2002, a bordo del B/O Francisco de Ulloa (Fig. 1). Se obtuvieron registros de conductividad, profundidad y temperatura. La salinidad fue calculada de acuerdo a los procedimientos estándar (UNESCO 1991), según una red de muestreo que contiene 17 estaciones, con la finalidad de obtener la descripción de las propiedades hidrográficas y la circulación geostrófica de la zona de estudio.

Para este manuscrito la bahía se ha dividido en 2 provincias: la profunda entre las estaciones (9-17) y la somera entre las estaciones (1-8). En la provincia profunda al noroeste y noreste de la bahía sobresalen la Cuenca Alfonso con la profundidad máxima $(450 \mathrm{~m})$ y la Boca Norte comunicación principal con el GC, con un umbral entre 250 y $300 \mathrm{~m}$, así como Canal San José con un umbral de $60 \mathrm{~m}$ en su comunicación con la bahía. En la provincia somera al suroeste y sureste destacan CSL enlace muy dinámico y somero con el GC y la Ensenada de La Paz, una laguna costera que cuenta con una importante infraestructura portuaria, urbana y turística, donde se localiza la Ciudad de La Paz, Capital del Estado de Baja California Sur, México (Fig. 1).

Con estos datos se realizaron perfiles verticales promedio de temperatura, salinidad y sigma-t. Para mostrar la distribución vertical de temperatura, salinidad y densidad, se utilizó la sección longitudinal (A-A', nortesur, estaciones 16 a 1), dividida en: la provincia profunda (estaciones 16,13 y 10 ) y la somera (estaciones 7,4 y 1 ) (Fig. 1).

Mediante el uso de diagramas TS se identificó la presencia de masas de agua en la región de estudio, de acuerdo a la clasificación de masas de agua de TorresOrozco (1993) y Castro et al. (2006). La cuantificación de la estratificación de la columna de agua se determinó a partir del cálculo del parámetro de estratificación $\phi$ (Joules por metro cúbico, $\mathrm{J} \mathrm{m}^{-3}$ ) (Simpson 1981), desde la superficie hasta los $100 \mathrm{~m}$ de profundidad, eliminando las estaciones con valores menores. 
Se calculó el Índice de Surgencia costera inducida por el viento expresado en $\left(\mathrm{m}^{3} \mathrm{~s}^{-1}\right)$ por $100 \mathrm{~m}$ de costa, para los vientos que soplan paralelos a la costa y que tienen posibilidad de generar una surgencia costera en la región de la costa occidental del Golfo de California (Silva \& Valdenegro 2003).

Para cuantificar la importancia de la estratificación, se calculó la frecuencia de Brunt-Väisälä $\left(\mathrm{s}^{-1}\right)$ interpretada como la frecuencia vertical excitada por un desplazamiento vertical de la parcela de fluido (Plata \& Filonov 2007).

La circulación geostrófica $\left(\mathrm{m} \mathrm{s}^{-1}\right)$ se obtuvo por análisis geostrófico estándar (Pond \& Pickard 1983 y Reiss et al. 2000).

\section{Resultados}

En la provincia profunda, durante mayo no se presentó una capa de mezcla vertical, con una temperatura superficial promedio (TSP) de $23^{\circ} \mathrm{C}$, con la máxima salinidad superficial promedio (SSP) $(35,37)$ de los 4 cruceros y una sigma-t superficial promedio (StSP) de $\left(24,21 \mathrm{~kg} \mathrm{~m}^{-3}\right)$, con un gradiente vertical térmico de $0,15^{\circ} \mathrm{C}$ $\mathrm{m}^{-1}$ (Tabla 1). Destaca la presencia de una termoclina intensa (Fig. 2a). La estructura de la haloclina se presentó bien definida y muy escarpada (Fig. 2b), con un gradiente halino de $\left(7,2910^{-3}\right.$ ups $\left.\mathrm{m}^{-1}\right)$ (Tabla 1$)$. Se encontró la picnoclina más escarpada de los 4 cruceros (Fig. 2c), con un gradiente de $\left(3,4110^{-2} \mathrm{~kg} \mathrm{~m}^{-3} \mathrm{~m}^{-1}\right)$, con un parámetro de estratificación $(\phi)$ promedio de $124,76 \mathrm{~J} \mathrm{~m}^{-3}$, la radiación solar promedio fue de $261,34 \mathrm{~W} \mathrm{~m}^{-2}$, con una evaporación de 271,69 mm, la precipitación fue de 1,50 mm y los vientos fueron débiles provenientes del sur de $3,50 \mathrm{~m} \mathrm{~s}^{-1}$, con una frecuencia de Brunt-Väisälä promedio de $1,2610^{-2} \mathrm{~s}^{-1}$, con el valor máximo a los $11 \mathrm{~m}$ de profundidad $\left(2,5910^{-2} \mathrm{~s}^{-1}\right) \mathrm{y}$ un índice de surgencia costera inducida por el viento de $59,52 \mathrm{~m}^{3} \mathrm{~s}^{-1}$ (Tabla 1). La isoterma de $\operatorname{los} 13^{\circ} \mathrm{C}$ marcó el fondo de la termoclina estacional en la Boca Norte, con la

Tabla 1. Comparación de valores de variables físicas, de la columna de agua, para mayo, julio, octubre de 2001 y febrero de 2002, en Bahía de La Paz, México / Comparison of values of physical variables of the water column, for May, July, October 2001 and February 2002 in Bahía de La Paz, Mexico

\begin{tabular}{|c|c|c|c|c|}
\hline Variable/Meses & Mayo/2001 & Julio/2001 & Octubre $/ 2001$ & Febrero/2002 \\
\hline Temperatura superficial promedio $\left({ }^{\circ} \mathrm{C}\right)$ & $23,00 \pm 0,86$ & $25,97 \pm 0,97$ & $27,88 \pm 0,26$ & $18,92 \pm 0,43$ \\
\hline Salinidad superficial promedio & $35,37 \pm 0,07$ & $35,22 \pm 0,04$ & $34,92 \pm 0,04$ & $35,35 \pm 0,02$ \\
\hline Sigma-t superficial promedio $\left(\mathrm{kg} \mathrm{m}^{-3}\right)$ & $24,21 \pm 0,25$ & $22,37 \pm 0,30$ & $22,37 \pm 0,09$ & $25,31 \pm 0,09$ \\
\hline Velocidad del viento $\left(\mathrm{m} \mathrm{s}^{-1}\right)$ & 3,50 & 2,70 & 4,10 & 5,20 \\
\hline Dirección del viento & Sur & Sur & Suroeste & Norte \\
\hline Capa de mezcla promedio (m) & ausencia & ausencia & 20 & 50 \\
\hline $\begin{array}{l}\text { Gradiente vertical de temperatura }\left({ }^{\circ} \mathrm{C} \mathrm{m}^{-1}\right) \text { a } 50 \mathrm{~m} \text { de } \\
\text { profundidad }\end{array}$ & $1,5410^{-1}$ & $1,7210^{-1}$ & $1,0110^{-1}$ & $9,9710^{-3}$ \\
\hline Gradiente vertical de salinidad a $50 \mathrm{~m}$ de profundidad & $7,2910^{-3}$ & $2,4910^{-3}$ & No aplica & $7,0210^{-4}$ \\
\hline $\begin{array}{l}\text { Gradiente vertical de sigma-t }\left(\mathrm{kg} \mathrm{m}^{-3} \mathrm{~m}^{-1}\right) \text { a } 50 \mathrm{~m} \text { de } \\
\text { profundidad }\end{array}$ & $3,4110^{-2}$ & $4,5910^{-2}$ & $2,9410^{-2}$ & $1,9910^{-3}$ \\
\hline Radiación solar recibida promedio $\left(\mathrm{W} \mathrm{m}^{-2}\right)$ & 261,34 & 241,86 & 216,01 & 193,85 \\
\hline Evaporación (mm) & 271,69 & 290,20 & 176,16 & 140,00 \\
\hline Precipitación (mm) & 1,50 & 71,40 & 0,00 & 12,50 \\
\hline Parámetro de estratificación $(\phi)$ promedio $\left(\mathrm{J} \mathrm{m}^{-3}\right)$ & 124,76 & 172,26 & 276,18 & 9,74 \\
\hline Frecuencia Brunt-Väisälä promedio $\left(\mathrm{s}^{-1}\right)$ & $1,2610^{-2}$ & $1,4510^{-2}$ & $1,3610^{-2}$ & $5,8510^{-3}$ \\
\hline Frecuencia Brunt-Väisälä máxima $\left(\mathrm{s}^{-1}\right)$ & $2,5910^{-2}$ & $2,9710^{-2}$ & $1,7710^{-2}$ & $7,2810^{-3}$ \\
\hline Índice de surgencia inducido por el viento $\left(\mathrm{m}^{3} \mathrm{~s}^{-1}\right)$ & 59,52 & 47,04 & 18,92 & No genera \\
\hline Esfuerzo del viento $\left(\mathrm{N} \mathrm{m}^{-2}\right)$ & $3,6910^{-2}$ & $1,3310^{-2}$ & $1,1710^{-2}$ & $4,9210^{-2}$ \\
\hline Velocidad geostrófica máxima superficial $\left(\mathrm{m} \mathrm{s}^{-1}\right)$ & 0,43 & 0,65 & 0,52 & 0,26 \\
\hline Velocidad geostrófica máxima a $50 \mathrm{~m}\left(\mathrm{~m} \mathrm{~s}^{-1}\right)$ & 0,12 & 0,30 & 0,15 & 0,18 \\
\hline Remolino geostrófico superficial & Ciclónico & Ciclónico & Ciclónico & Anticiclónico \\
\hline Remolino geostrófico a $50 \mathrm{~m}$ & Ciclónico & Ciclónico & Ciclónico & Anticiclónico \\
\hline
\end{tabular}



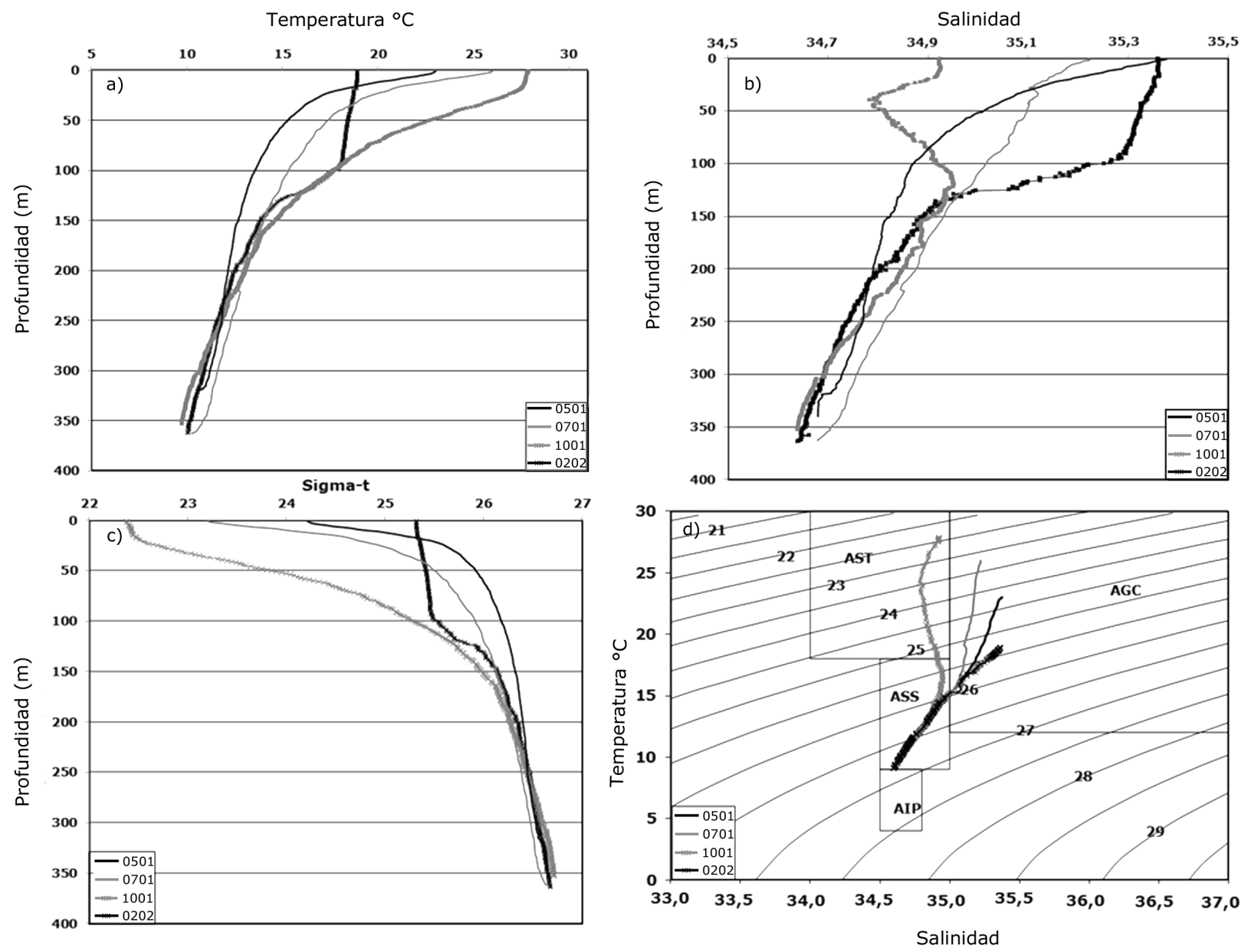

Figura 2. Perfiles verticales promedio durante mayo, julio, octubre de 2001 y febrero de 2002. (a) Temperatura en ${ }^{\circ} \mathrm{C}$, (b) salinidad, (c) sigmat en $\mathrm{kg} \mathrm{m}^{-3}$ y (d) masas de agua. AGC (Agua del Golfo de California), AST (Agua Superficial Tropical), ASS (Agua Subsuperficial Subtropical) y AIP (Agua Intermedia del Pacífico) / Average vertical profiles during May, July, October 2001 and February 2002. (a) Temperature in ${ }^{\circ} \mathrm{C}$, (b) salinity, (c) sigma-t in $\mathrm{kg} \mathrm{m}^{-3}$ and (d) water masses. AGC (Gulf of California Water), AST (Tropical Surface Water), ASS (Subtropical Subsurface Water) and AIP (Pacific Intermediate Water)

mayor estratificación térmica hasta los $50 \mathrm{~m} \mathrm{de}$ profundidad. Se registró la elevación de las isotermas desde los $225 \mathrm{~m}$ entre las estaciones E16 y E10, con una diferencia de temperatura de $12^{\circ} \mathrm{C}$ entre la superficie y el fondo (Fig. 3a). La isohalina de los 34,90 marcó el fondo de la haloclina estacional, con la mayor estratificación halina hasta los $80 \mathrm{~m}$ de profundidad. Se aprecian las isohalinas elevadas desde los $200 \mathrm{~m}$ alrededor de la estación E13, donde es evidente el montículo de la isohalina de 34,80 (Fig. 4a). También se aprecia la elevación de las isopicnas desde los $200 \mathrm{~m}$ alrededor de la estación E13. La isopicna de los $26,20 \mathrm{~kg} \mathrm{~m}^{-3}$ marcó el fondo de la picnoclina estacional, con el máximo gradiente en los primeros $50 \mathrm{~m}$ de profundidad (Fig. 5a). Según el diagrama T-S, en mayo en la provincia profunda se registró AGC (S $\geq 35, \mathrm{~T} \geq 12^{\circ} \mathrm{C}$ ), en una capa de $51 \mathrm{~m}$ de espesor, el resto de la columna presentó características del ASS $(34,50 \leq \mathrm{S}$ $\leq 35,9 \leq \mathrm{T} \leq 18^{\circ} \mathrm{C}$ ) (Fig. 2d). La distribución espacial de las velocidades geostróficas en la provincia profunda en mayo, muestra la entrada de agua por la Boca Norte frente a Cabeza de Mechudo con una salida frente al complejo insular Espíritu Santo-La Partida y el inicio de una circulación superficial de tipo ciclónica con una velocidad máxima de $0,43 \mathrm{~m} \mathrm{~s}^{-1}$ (Tabla 1), que se hace evidente a los $50 \mathrm{~m}$ de profundidad, donde continúa la entrada de agua del golfo (Figs. 6a y 7a). 

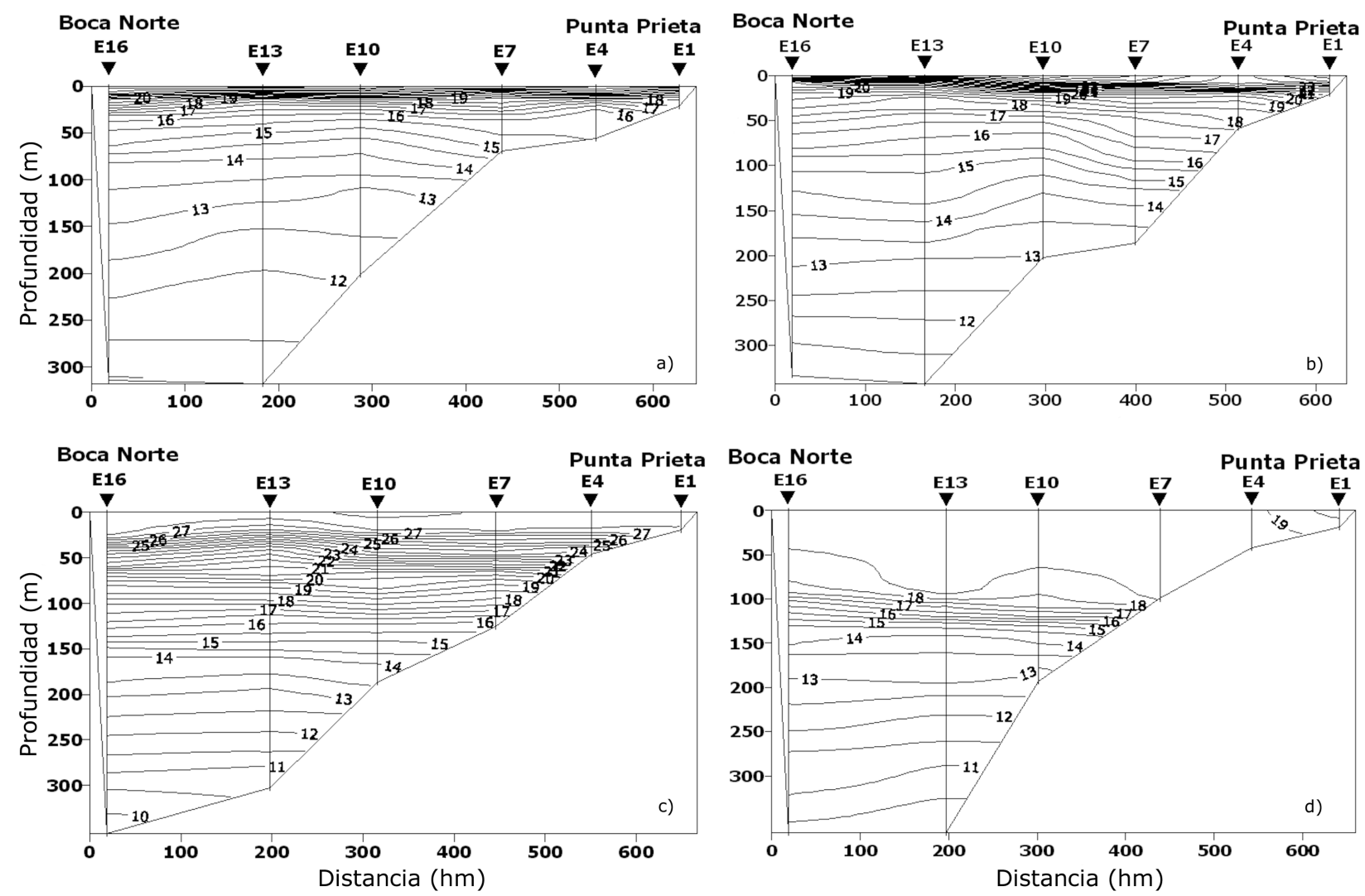

Figura 3. Sección longitudinal de temperatura en ${ }^{\circ} \mathrm{C}$ durante: mayo (a), julio (b) y octubre (c) de 2001 y febrero (d) de 2002 / Longitudinal section of temperature in ${ }^{\circ} \mathrm{C}$ during: May (a), July (b) and October (c) 2001 and February (d) 2002

Durante julio continuó ausente la capa de mezcla en la bahía, la TSP se incrementó a $25,97^{\circ} \mathrm{C}$, la SSP disminuyó a 35,22 , consecuentemente la StSP disminuyó a $(22,37 \mathrm{~kg}$ $\mathrm{m}^{-3}$ ), con un gradiente vertical térmico que se incrementó a $0,17^{\circ} \mathrm{C} \mathrm{m}^{-1}$ con respecto a mayo (Tabla 1 ). Toda la columna se registró más caliente, con la termoclina estacional más intensa con respecto a mayo (Fig. 2a). Nuevamente se apreciaron las isotermas elevadas desde los $90 \mathrm{~m}$ en la región profunda alrededor de la estación E13, con una estratificación térmica notable en los primeros $100 \mathrm{~m}$ de profundidad (Fig. 3b). Se registró una disminución gradual de la salinidad, desde la superficie hasta los $363 \mathrm{~m}$ de profundidad $(34,67)$, sin la presencia de la haloclina (Fig. 2b), con un gradiente de $\left(2,4910^{-3}\right.$ ups $\mathrm{m}^{-1}$ ) (Tabla 1). Sobresale la elevación de la isohalina de 35,05 alrededor de la estación E10, así como una importante parcela de esta agua, que ocupó la mayor parte de la columna entre los 5 y $80 \mathrm{~m}$ de profundidad (Fig. 4b). Destaca una picnoclina muy inclinada, más profunda que en mayo, con una densidad menor en toda la columna (Fig. 2c), con un gradiente vertical de $\left(4,5910^{-2} \mathrm{~kg} \mathrm{~m}^{-3} \mathrm{~m}^{-1}\right)$ y una estratificación $(\phi)$ promedio que se incrementó en relación a mayo a $172,26 \mathrm{~J} \mathrm{~m}^{-3}$. La radiación solar promedio mensual (julio) fue de $241,86 \mathrm{~W} \mathrm{~m}^{-2}$, con una evaporación de 290,20 mm, la precipitación fue de 71,40 mm y los vientos fueron débiles provenientes del sur de $2,70 \mathrm{~m} \mathrm{~s}^{-1}$, con una frecuencia de Brunt-Väisälä promedio de $1,4510^{-2}$ $\mathrm{s}^{-1}$, con el valor máximo a los $14 \mathrm{~m}$ de profundidad $(2,97$ $10^{-2} \mathrm{~s}^{-1}$ ) y un índice de surgencia costera inducida por el viento de $47,04 \mathrm{~m}^{3} \mathrm{~s}^{-1}$ (Tabla 1). Resalta en la provincia profunda la elevación de las isopicnas desde los $90 \mathrm{~m}$ de profundidad alrededor de la estación E13, alternando con el hundimiento de las isopicnas alrededor de la estación E10 desde la superficie hasta los $50 \mathrm{~m}$ de profundidad 

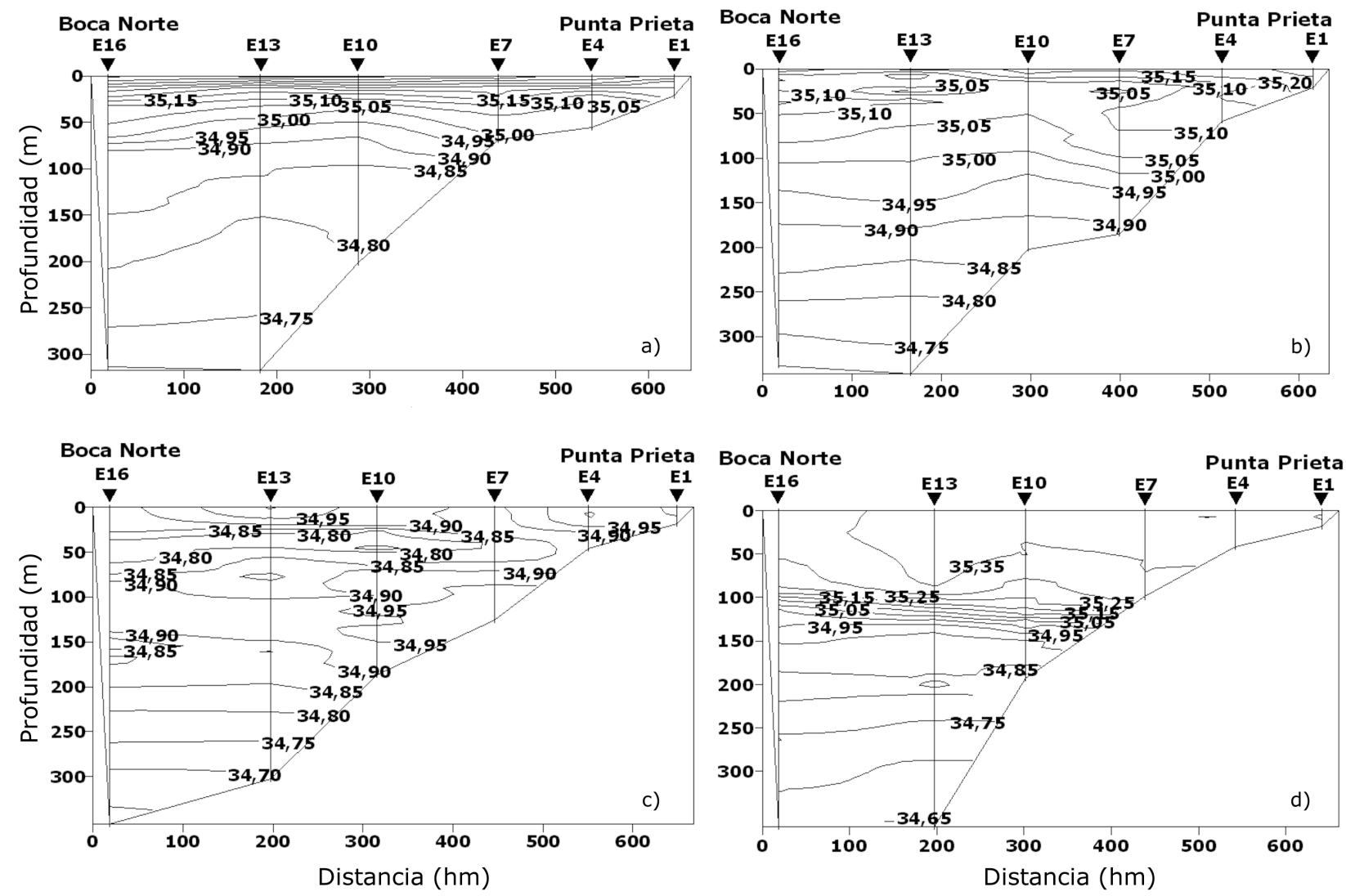

Figura 4. Sección longitudinal de salinidad durante: mayo (a), julio (b) y octubre (c) de 2001 y febrero (d) de 2002 / Longitudinal section of salinity during: May (a), July (b) and October (c) 2001 and February (d) 2002

(Fig. 5b). En julio, aumentó la presencia del AGC, con una capa de 109 m de espesor; a mayor profundidad se registró solamente ASS (Fig. 2d). En julio la circulación superficial termohalina de tipo ciclónica se hizo más intensa y se centró sobre la provincia profunda de la bahía, con la entrada de agua por la Boca Norte frente a Cabeza de Mechudo con una velocidad máxima de $0,65 \mathrm{~m} \mathrm{~s}^{-1}$ (Tabla 1), entretanto a los $50 \mathrm{~m}$ de profundidad prevalece la estructura ciclónica, con agua saliendo por la Boca Norte frente a la costa insular Espíritu Santo - La Partida que se dirige hacia la Isla San José (Figs. 6b y 7b).

En octubre, se detectó una capa de mezcla promedio de $20 \mathrm{~m}$ de profundidad con base al criterio (Levitus 1982), registrándose la TSP máxima $\left(27,88^{\circ} \mathrm{C}\right)$, así como la menor SSP $(34,92)$ y la mínima StSP $\left(22,37 \mathrm{~kg} \mathrm{~m}^{-3}\right)$ de los 4 cruceros, con un gradiente térmico de $1,0110^{-10} \mathrm{C} \mathrm{m}^{-1}$ (Tabla 1). La termoclina continuó su proceso de hundimiento con respecto a mayo, apareciendo prominente, abajo de la capa de mezcla y hasta los $160 \mathrm{~m}$ de profundidad (Fig. 2a). La elevación de las isotermas se aprecia más localizada y pronunciada que en mayo y julio desde los $200 \mathrm{~m}$ de profundidad, alrededor de la estación E13, con mayor estratificación térmica en toda la columna (Fig. 3c). En la estructura de la salinidad, destaca una disminución desde la superficie hasta los $40 \mathrm{~m}$ de profundidad con salinidad de 34,78 , seguida de un incremento (hasta 34,95 ) a los 120 $\mathrm{m}$ de profundidad. Es de resaltar, que está salinidad fue mayor que la salinidad superficial $(34,92)$ (Tabla 1), después de esta profundidad decrece de manera relativamente gradual (Fig. 2b). Se distingue una lengüeta de agua con salinidad de 34,80 presente entre los 36 y los $60 \mathrm{~m}$ de profundidad, entre capas de mayor salinidad (Fig. 4c). Con una picnoclina prominente después de la capa de mezcla, hasta los $160 \mathrm{~m}$ de profundidad y la menor 

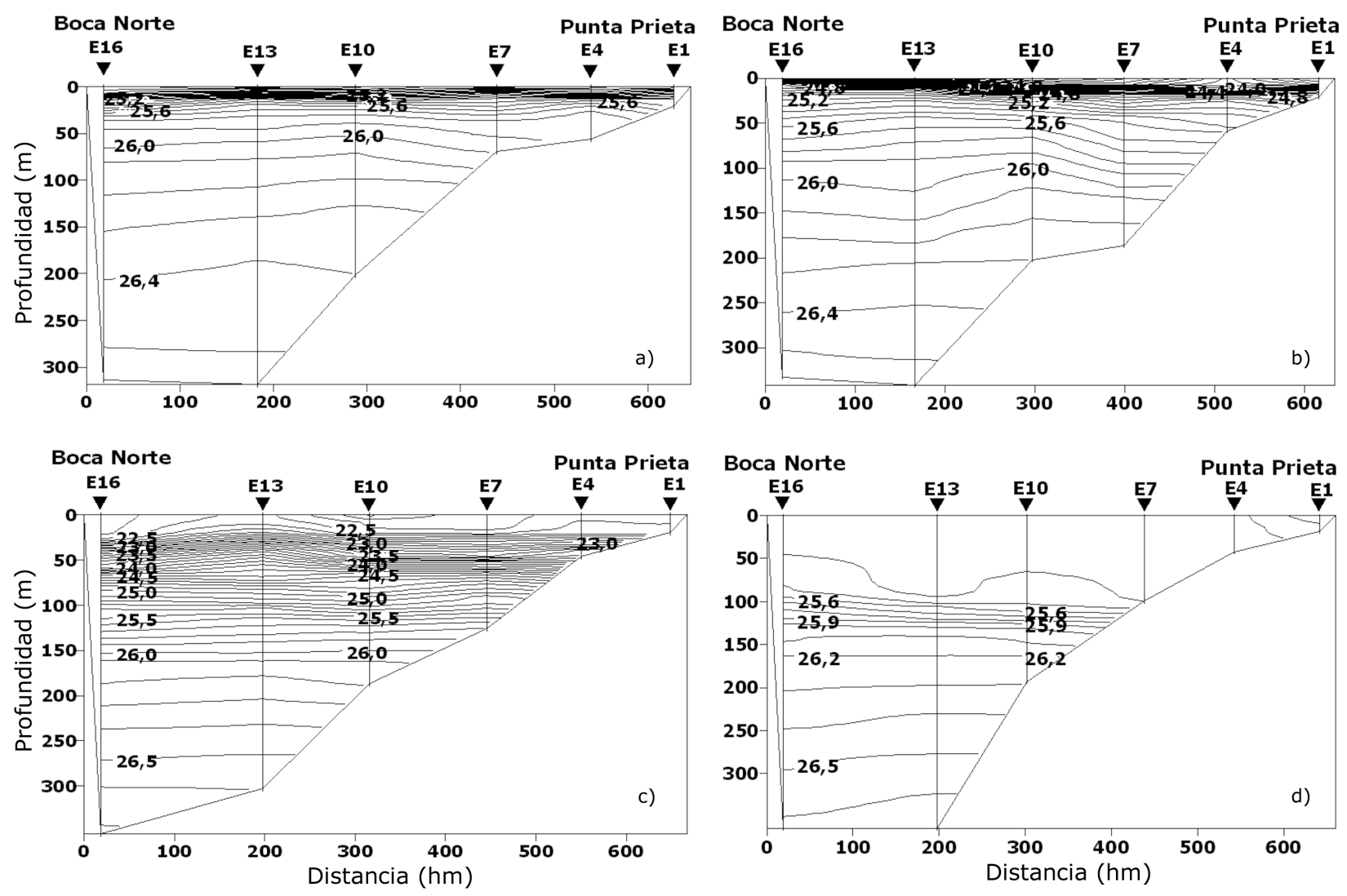

Figura 5. Sección longitudinal de sigma-t en $\mathrm{kg} \mathrm{m}^{-3}$ durante: mayo (a), julio (b) y octubre (c) de 2001 y febrero (d) de 2002/ Longitudinal section of sigma-t in $\mathrm{kg} \mathrm{m}^{-3}$ during: May (a), July (b) and October (c) 2001 and February (d) 2002

densidad de los 4 cruceros hasta los $215 \mathrm{~m}$ de profundidad (Fig. 2c). Con un gradiente de $2,9410^{-2} \mathrm{~kg} \mathrm{~m}^{-3} \mathrm{~m}^{-1}$, con la estratificación promedio máxima $\left(276,18 \mathrm{~J} \mathrm{~m}^{-3}\right)$ de este estudio, la radiación solar promedio mensual (octubre) fue de 216,01 $\mathrm{W} \mathrm{m}^{-2}$, con una evaporación de 176,16 mm, no se presentó precipitación y los vientos fueron del suroeste de $4,10 \mathrm{~m} \mathrm{~s}^{-1}$, con una frecuencia de BruntVäisälä promedio de $1,3610^{-2} \mathrm{~s}^{-1}$, con el valor máximo a los $63 \mathrm{~m}$ de profundidad $\left(1,7710^{-2} \mathrm{~s}^{-1}\right)$ y un índice de surgencia costera inducida por el viento de $18,92 \mathrm{~m}^{3} \mathrm{~s}^{-1}$ (Tabla 1). Se aprecian elevadas las isopicnas desde $\operatorname{los} 238 \mathrm{~m} \mathrm{de}$ profundidad en la provincia profunda alrededor de la estación E13, con la isopicna de $26 \mathrm{~kg} \mathrm{~m}^{-3}$ marcando el fondo de la picnoclina estacional (Fig. 5c). En octubre, no se registró AGC, en su lugar se encontró Agua Superficial Tropical (AST; $\mathrm{S}<35, \mathrm{~T} \geq 18^{\circ} \mathrm{C}$ ), desde la superficie hasta los $95 \mathrm{~m}$ de profundidad, después de esta profundidad se halló exclusivamente ASS, hasta los
353 m (Figs. 2d y 4c). En octubre, también se encontró una circulación con estructura ciclónica, con la entrada del agua desde el golfo frente a Cabeza de Mechudo y la salida por la costa oeste del complejo insular Espíritu Santo-La Partida con una velocidad máxima de $0,52 \mathrm{~m} \mathrm{~s}^{-1}$ (Tabla 1), esta circulación prevaleció hasta los $50 \mathrm{~m}$ de profundidad y parte del agua de reflujo se integra a un remolino anticiclónico frente a la Boca Norte (Figs. 6c y 7c).

En febrero, se midió la capa de mezcla promedio máxima de los 4 cruceros (50 $\mathrm{m}$ de profundidad), con base al criterio (Levitus 1982). Se registró la TSP mínima $\left(18,92^{\circ} \mathrm{C}\right)$, con una SSP de 35,35 y la mayor $\operatorname{StSP}\left(25,31 \mathrm{~kg} \mathrm{~m}^{-3}\right)$ de este estudio (Tabla 1). Con un drástico hundimiento de la termoclina (hasta $100 \mathrm{~m}$ en algunas estaciones), con la consecuente disminución del grosor de dicha termoclina que se localizó entre los 100 y 160 m de profundidad (Fig. 

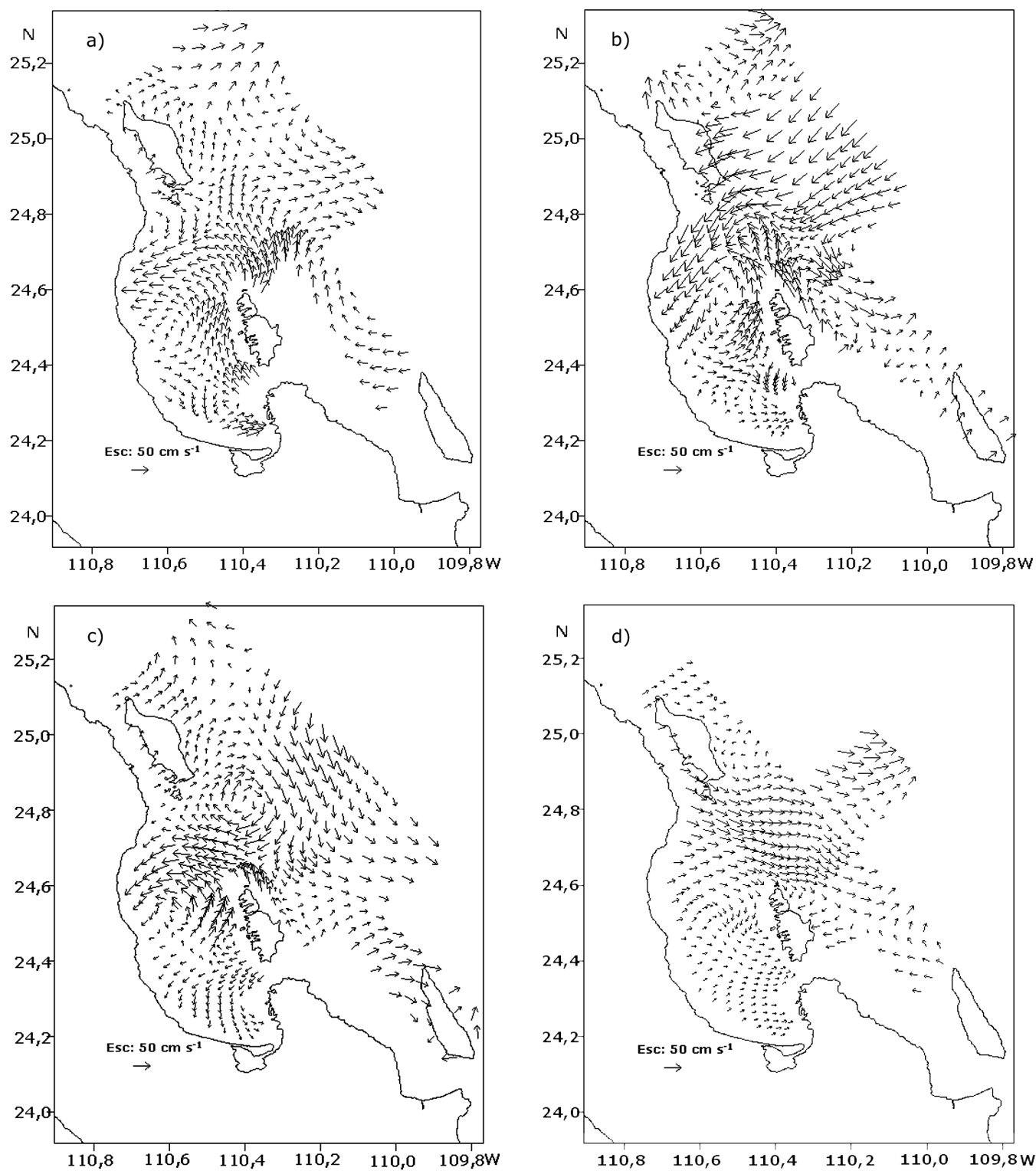

Figura 6. Velocidades geostróficas superficiales $\left(\mathrm{m} \mathrm{s}^{-1}\right)$ durante: mayo (a), julio (b) y octubre (c) de 2001 y febrero (d) de 2002 / Geostrophic surface velocities ( $\mathrm{m} \mathrm{s}^{-1}$ ) during: May (a), July (b) and October (c) 2001 and February (d) 2002

2a), con un gradiente de $\left(9,9710^{-30} \mathrm{C} \mathrm{m}^{-1}\right)$ (Tabla 1). Las aguas en la provincia profunda se encontraron frías y con una capa de mezcla excepcional que se hunde desde los $42 \mathrm{~m}$ en la Boca Norte hasta los $94 \mathrm{~m}$ alrededor de la estación E13. Después de la capa de mezcla toda la columna de agua se encontró estratificada y la isoterma de los $10,50^{\circ} \mathrm{C}$ marcó el fondo de dicha estratificación. En la Boca
Norte se aprecia el hundimiento de la isoterma de los $18,50^{\circ} \mathrm{C}$, marcando el límite de la capa de mezcla, desde los $42 \mathrm{~m}$ hasta los $94 \mathrm{~m}$ alrededor de la estación E13 (Fig. $3 d)$. Se registró la mayor salinidad de los 4 cruceros hasta los $128 \mathrm{~m}$, excepto en los dos primeros metros (Fig. 2b), la

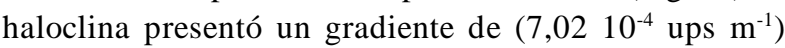
(Tabla 1). La isohalina de los 35,35 marca el fondo de la 

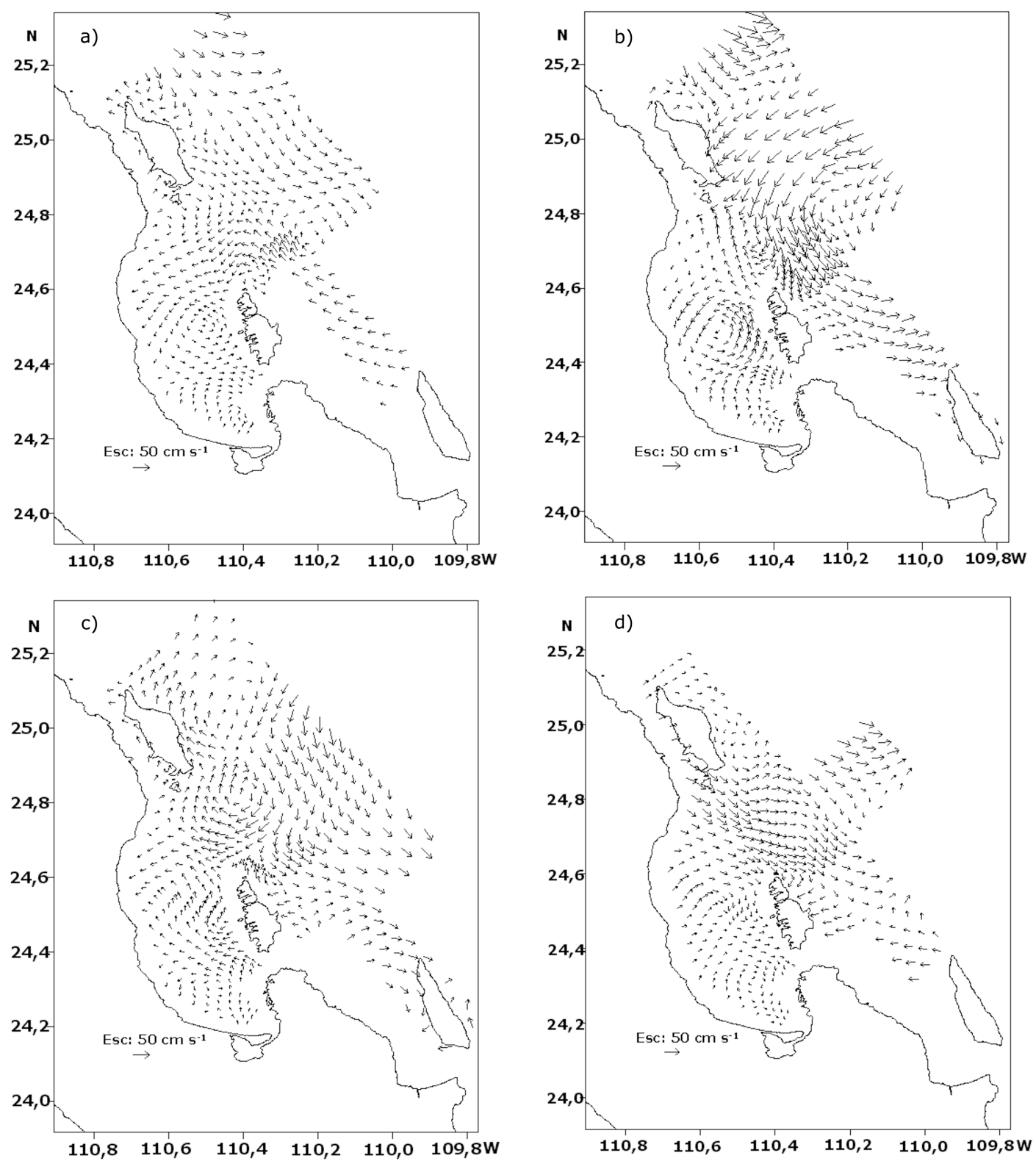

Figura 7. Velocidades geostróficas ( $\mathrm{m} \mathrm{s}^{-1}$ ) a $\mathbf{5 0 ~} \mathrm{m}$ de profundidad durante: mayo (a), julio (b) y octubre (c) de 2001 y febrero (d) de 2002 / Geostrophic velocities ( $\mathrm{m} \mathrm{s}^{-1}$ ) at $50 \mathrm{~m}$ depth during: May (a), July (b) and October (c) 2001 and February (d) 2002

capa de mezcla, que afloró en la Boca Norte, después se hundió hasta los $87 \mathrm{~m}$ en la estación E13 y luego se elevó hasta los $37 \mathrm{~m}$ alrededor de la estación E10, para nuevamente hundirse hasta $\operatorname{los} 70 \mathrm{~m}$ en la región intermedia E7, con la isohalina de 34,90 marcando el fondo de la haloclina (Fig. 4d). La picnoclina se suavizó y se hundió hasta los $170 \mathrm{~m}$ de profundidad aproximadamente
(Fig. 2c), con un gradiente de 1,99 $10^{-3} \mathrm{~kg} \mathrm{~m}^{-3} \mathrm{~m}^{-1}$ (Tabla 1), la estratificación promedio disminuyó de manera drástica, y fue la menor de los 4 cruceros con $9,74 \mathrm{~J} \mathrm{~m}^{-3}$, la radiación solar promedio mensual (febrero) fue de 193,85 $\mathrm{W} \mathrm{m}^{-2}$, con una evaporación de $140 \mathrm{~mm}$, con una precipitación de 12,50 mm y los vientos fueron del norte de $5,20 \mathrm{~m} \mathrm{~s}^{-1}$, con una frecuencia de Brunt-Väisälä promedio de 5,85 10 
$\mathrm{s}^{-1}$, con el valor máximo a los $153 \mathrm{~m}$ de profundidad $(7,28$ $10^{-3} \mathrm{~s}^{-1}$ ) (Tabla 1). La isopicna de los $25,40 \mathrm{~kg} \mathrm{~m}^{-3}$ se hundió desde los 46 m en la Boca Norte en la estación E16 hasta los $94 \mathrm{~m}$ en la provincia profunda alrededor de la estación E13 y luego se elevó hasta los 63 m en la estación E10, con la isopicna de los $26,20 \mathrm{~kg} \mathrm{~m}^{-3}$ marcando el límite de la picnoclina (Fig. 5d). Para febrero, se registró de nuevo AGC, desde la superficie hasta los $125 \mathrm{~m}$ de profundidad en la provincia profunda. A mayor profundidad y hasta los $364 \mathrm{~m}$ se encontró ASS (Fig. 2d). La distribución termohalina de febrero, dio origen a una circulación superficial que muestra el agua saliendo de la bahía por la Boca Norte, con el inició de una circulación con forma anticiclónica con una velocidad máxima de $0,26 \mathrm{~m} \mathrm{~s}^{-1}$ (Tabla 1), tal comportamiento predominó hasta los $50 \mathrm{~m}$ de profundidad (Figs. 6d y 7d).

\section{Discusión}

La estratificación y la abrupta termoclina, haloclina y picnoclina en mayo y la escarpada termoclina y picnoclina en julio, fueron causadas principalmente por el arribo del agua del golfo fría y densa, producto de la flotabilidad positiva originada por la surgencia costera que se genera en esta época del año en la costa occidental del GC, debido a la acción de los vientos del sur y la mayor radiación solar recibida. Mientras que la disminución de la estratificación salina y la ausencia de la haloclina en julio, fue debido a la homogenización halina y al incremento del AGC en la bahía.

El papel de la surgencia impulsada por el viento en la estratificación de una bahía semiárida en el GC se ha explicado con observaciones en Bahía Concepción, Baja California Sur, México. La estratificación en Bahía Concepción está relacionada con la transferencia de calor estacional desde la atmósfera, así como a las intrusiones de agua fría forzadas por la surgencia impulsada por el viento. Al inicio del verano, la surgencia de los vientos causó la intrusión de agua fría que bajó la temperatura en la columna de agua. Los vientos persistentes favorecen la surgencia manteniendo el suministro de agua fría en la bahía, lo que aumentó la estratificación de la temperatura cuando el agua superficial aumenta su temperatura (Cheng et al. 2010).

En verano en el GC, los vientos superficiales resultantes soplan desde el sur, generando surgencia en las costas de la península de Baja California (BadanDangon et al. 1991, Lavín et al. 1997 y Bernal et al. 2001). Durante esta estación se intensifica la Corriente
Norecuatorial y el desarrollo de la Corriente de Costa Rica acarrea agua subtropical hacia el golfo (Baumgartner \& Christensen 1985 y Badan-Dangon 1998).

En relación con esta condición de BLP, Jiménez-Illescas et al. (1994) con datos de junio de 1992, encontraron la termoclina en la superficie, con una fuerte estratificación entre 25 y 50 m, Monreal-Gómez et al. (2001) con un muestreo en junio de 1998 identificaron 3 masas de agua en la bahía AGC, ASE y ASS y Salinas-González et al. (2003) procesando datos de 3 campañas oceanográficas, caracterizaron la estructura termohalina de la parte profunda de la bahía, determinando que durante el verano, se desarrolla una intensa estratificación desde la superficie.

La gran estratificación de temperatura y densidad en octubre, se produjo por la condición local de radiación solar y la intrusión de agua caliente proveniente de la boca del golfo, mientras que la capa de mezcla registrada, fue generada por los vientos en la bahía y en la zona adyacente del golfo. Entretanto, la estructura y disminución de la salinidad con un mínimo y un máximo subsuperficial, debió ser causada por la entrada de agua con menor salinidad del golfo $(\mathrm{S}<35)$ proveniente al parecer del Pacífico Tropical, que sustituyó al AGC con salinidad ( $\mathrm{S} \geq 35$ ) en esta bahía, considerada una cuenca de evaporación, sin descargas fluviales, con evaporación de $176,16 \mathrm{~mm}$ y precipitación nula en este mes de octubre.

La importante capa de mezcla en el perfil vertical térmico, de salinidad y de densidad en febrero, así como la significante disminución de la estratificación, fue el resultado de la homogenización y el enfriamiento de la columna de agua por los vientos del norte en la región y la menor radiación solar recibida.

En invierno, se generan vientos intensos en el GC en dirección noroeste-sureste. Estos vientos generan la surgencia más importante del GC en las costas de Sonora y Sinaloa con una pérdida de calor de la capa de mezcla que, junto con la menor insolación, se manifiestan como una disminución de la temperatura superficial del mar en esta época del año (Badan-Dangon et al. 1991 y Bernal et al. 2001).

En circunstancia similar en BLP, Jiménez-Illescas (1996) realizó el análisis de procesos barotrópicos y baroclínicos en la bahía con datos de marzo de 1994, no identificó masas de agua diferentes, en cuanto al viento si es persistente e intenso $\left(\geq 7 \mathrm{~m} \mathrm{~s}^{-1}\right)$ impulsa corrientes en las zonas poco profundas y principalmente en los canales, 
llegando a dominar el patrón de circulación. A su vez, Guevara-Guillén (2011), comparando datos de 4 cruceros en el 2009, reportó importantes variaciones espaciales y temporales de la hidrografía en la bahía y su zona oceánica adyacente. Por tanto, la bahía se destacó por mantener un retraso respecto a las condiciones presentes en el sur del GC.

La temperatura de la capa superficial (hasta los $15 \mathrm{~m}$ de profundidad) en la bahía presentó una variación clara de tipo estacional. Se registró un incremento gradual de mayo a julio con el valor máximo en octubre, mientras que para febrero se registró la menor temperatura. Esto fue originado por la condición local de la radiación solar recibida y la velocidad de los vientos en la zona, que dominaron la influencia de las aguas del golfo.

El enfriamiento anómalo que se presentó entre los 15 y $141 \mathrm{~m}$ de profundidad en los cruceros de mayo y julio, se debió a la entrada de agua fría del golfo, producto de la surgencia costera generada por la acción de los vientos del sur en la región.

Según el diagrama TS, las aguas con temperaturas mayores a $15^{\circ} \mathrm{C}$, presentaron una variabilidad considerable en las propiedades termohalinas y en sigma-t, contrastando con las aguas de temperaturas menores que mostraron características relativamente uniformes. El intercambio de agua entre la bahía y el GC implicó, en 3 de los cruceros (mayo, julio y febrero), exclusivamente AGC en la parte superficial (hasta los $100 \mathrm{~m}$ ) y ASS en la parte profunda, mientras que en octubre, de manera excepcional el AST sustituyó al AGC, acompañándola en la porción profunda el ASS. El umbral batimétrico de la Boca Norte, (de $250 \mathrm{~m}$ de profundidad), impidió la incursión del Agua Intermedia del Pacífico que está presente en la región adyacente del GC (Castro et al. 2006). Este umbral batimétrico a lo largo de Boca Norte al parecer, también puede bloquear la salida del agua profunda al GC, lo que podría contribuir a disminuir el contenido de oxígeno en el agua profunda de la bahía.

Durante los muestreos de mayo y julio, el AGC se localizó en la región de la termoclina-picnoclina, mientras que en febrero se ubicó en la capa de mezcla y en la zona de la termoclina-picnoclina. Entretanto, en octubre se localizó únicamente AST en la capa de mezcla y en la región de la termoclina-picnoclina. Por debajo de la termoclina-picnoclina, se encontró solamente ASS en los 4 cruceros (mayo, julio, octubre y febrero).
El calentamiento solar conservó la capa superficial caliente durante mayo, julio y octubre $\left(23,25,97\right.$ y $27,88^{\circ} \mathrm{C}$ respectivamente). En febrero, la mezcla inducida por el esfuerzo del viento, profundizó esta energía térmica desde la superficie hasta $\operatorname{los} 100 \mathrm{~m}$ de profundidad en algunas estaciones. Como consecuencia de ello, las termoclinas, haloclinas y picnoclinas se localizaron después de esta profundidad.

Las termoclinas y la picnoclinas afloraron en mayo y julio, se profundizaron en octubre y obtuvieron su máxima profundidad en febrero. Se registraron termoclinas y picnoclinas muy bien definidas, en mayo, julio y octubre, cuando presentaron un gradiente vertical empinado.

La estratificación térmica y de densidad, pueden impedir la mezcla vertical entre la capa superficial y las aguas profundas, lo que podría generar que el contenido de oxígeno sea pobre por debajo de la termoclinapicnoclina y que la renovación de los nutrientes tienda a disminuir en la superficie.

En mayo, julio y octubre, la circulación baroclínica en la provincia profunda de la bahía, estuvo dominada por un remolino ciclónico, que facilitó el intercambio del agua superficial y subsuperficial con el golfo, con las velocidades máximas en julio en la superficie y a los $50 \mathrm{~m}$ de profundidad. El domo de las isotermas e isopicnas relacionado con esta circulación, se presentaron a diferentes profundidades y en diferentes posiciones en dicha provincia.

Las características de la provincia profunda son aplicables a la zona de transiciones de la bahía, mientras que la provincia somera tiene un comportamiento propio. En mayo y julio la columna estuvo completamente estratificada en temperatura, salinidad y densidad. Contrastando con febrero, cuando se encontró una homogenización total térmica, halina y en densidad. Para octubre, se presentó un comportamiento intermedio, con una capa de mezcla somera y el resto de la columna bien estratificada térmicamente y en densidad, entretanto la salinidad mostró una estratificación relativamente débil.

En mayo y julio, la mayor parte del agua presentó características del AGC, con la intrusión en el fondo del ASS hasta frente de la Punta Sur de la Isla Espíritu Santo (PSIES). Diferenciando en octubre cuando el AST ocupó la provincia somera, con la intrusión del ASS en el fondo hasta frente de la PSIES y trazas de AGC, que al parecer es la firma de la descarga del agua de la Ensenada de la Paz, donde la evaporación aumentó la salinidad, debido a que es muy somera ( $3 \mathrm{~m}$ en promedio). 
La circulación baroclínica en la provincia somera se caracterizó por un remolino ciclónico superficial y un anticiclónico subsuperficial en mayo, julio y octubre, mientras que en febrero solamente se presentó un remolino anticiclónico subsuperficial y la salida del agua superficial por CSL.

Se encontró variabilidad espacial en temperatura, densidad, salinidad, estratificación y circulación baroclínica en BLP durante el período de ENSO neutral muestreado, así como la presencia de diferentes masas de agua en la región, con marcada diferencia entre la provincia profunda y la provincia somera, lo que proporciona una contribución a la oceanografía regional.

\section{Agradecimientos}

El presente estudio fue realizado con datos obtenidos a través del proyecto CONACYT 34071-T y del proyecto CGPI-20018002, financiado por el Instituto Politécnico Nacional dirigidos por la Dra. Laura Sánchez Velasco. Se agradece al Dr. Bernardo Shirasago Germán jefe de crucero y a los estudiantes del programa de Maestría en Manejo de Recursos Marinos del CICIMAR-IPN, por su participación en los 4 cruceros denominados BAGOL. Agradecemos también al CICESE y a la tripulación del B/ O Francisco de Ulloa por su valioso apoyo.

\section{LITERATURA CITADA}

Badan-Dangon A. 1998. Coastal circulation from the Galapagos to the Gulf of California. In: Robinson AR \& KH Brink (eds). The Sea 11: 315-343. John Wiley and Sons, New York.

Badan-Dangon A, CE Dorman, MA Merrifield \& CD Winant. 1991. The lower atmosphere over the Gulf of California. Journal of Geophysical Research 96(C9): 16877-16896.

Baumgartner RT \& N Christensen. 1985. Coupling of the Gulf of California to large-scale interannual climatic variability. Journal of Marine Research 43: 825-848.

Bernal G, P Ripa \& JC Herguera. 2001. Variabilidad oceanográfica y climática en el bajo Golfo de California: influencia del Trópico y Pacífico Norte. Ciencias Marinas 27(4): 595-617.

Bustillos-Guzman J. 1995. Specific phytoplankton signatures and their relationship to hydrographic conditions in the coastal north-western Mediterranean Sea. Marine Ecology Progress Series 124: 247-258.

Castro R, R Durazo, A Mascarenhas, CA Collins \& A Trasviña. 2006. Thermohaline variability and geostrophic circulation in the southern portion of the Gulf of California. Deep-Sea Research I 53: 188-200.
Cheng P, A Valle-Levinson, CD Winant, ALS Ponte, G Gutierrez-Velasco \& KB Winters. 2010. Upwellingenhanced seasonal stratification in a semiarid bay. Continental Shelf Research 30: 1241-1249.

Douglas MW, RA Maddox, K Howard \& S Reyes. 1993. The Mexican monsoon. Journal of Climate 6: 1665-1677.

Falkowski GP \& JA Raven. 1997. Aquatic photosynthesis, 375 pp. Blackwell Science, Oxford.

Fiedler PC. 1986. Offshore entrainment of anchovy spawning habitat, eggs, and larvae by a displaced eddy in 1985 . California Cooperative Oceanic Fisheries Investigations Reports 17: 144-152.

García E. 1973. Modificaciones al sistema de clasificación de Koopen, 246 pp. Instituto de Geofísica, Universidad Nacional Autónoma de México, México.

Govoni JJ. 1993. Flux of larval fishes across frontal boundaries: examples from the Mississippi river plume front and the western Gulf Stream front in winter. Bulletin of Marine Science 53: 538-566.

Guevara-Guillén C. 2011. Hidrografía en bahía de La Paz y zona oceánica adyacente (2009). Tesis de Maestría, Centro Interdisciplinario de Ciencias Marinas, IPN, La Paz, 152 pp.

Hinojosa-Larios JA. 2007. Caracterización de los perfiles verticales de clorofila a en una bahía subtropical del Pacífico mexicano. Tesis de Maestría, Centro Interdisciplinario de Ciencias Marinas, IPN, La Paz, 104 pp.

INEGI. 1994. Aspectos geográficos de Baja California Sur, 30 pp. Instituto Nacional de Estadística, Geografía e Informática, México.

Jiménez-Illescas AR. 1996. Análisis de procesos barotrópicos y baroclínicos en la Bahía de La Paz, BCS. Tesis Doctoral, Universidad Nacional Autónoma de México, México, 212 pp.

Jiménez-Illescas AR, M Obeso-Nieblas \& MA AlatorreMendieta. 1994. Hidrología de la Bahía de La Paz. Oceanología, Unidad de Educación en Ciencia y Tecnología de Mar 1: 115-131.

Jiménez-Illescas AR, M Obeso-Nieblas \& DA Salas de L. 1997. Oceanografía física de la Bahía de La Paz, BCS. En: Urbán RJ \& M Ramírez (eds). La Bahía de La Paz, Investigaciones y conservación, pp. 31-41. Universidad Autónoma de Baja California Sur, Centro Interdisciplinario de Ciencias Marinas, Scripp Institute Oceanographic, La Paz.

Lavín MF, E Beier \& A Badan. 1997. Estructura hidrográfica y circulación del Golfo de California: Escalas estacional e interanual. En: Lavín MF (ed). Contribuciones a la oceanografía física en México. Monografía 3: 141-171. Unión Geofísica Mexicana, Ensenada. 
Levitus S. 1982. Climatological atlas of the world ocean, NOAA Professional Paper 13: 1-173.

Millán-Núñez R, R Cajal-Medrano, E Santamaría-Del Ángel \& E Millán-Núñez. 1993. Productividad primaria y clorofila $a$ en la parte central del golfo de California (otoño 1987). Ciencias Marinas 19(1): 29-40.

Monreal-Gómez MA, A Molina-Cruz \& DA Salas-de-León. 2001. Water masses and cyclonic circulation in Bay of $\mathrm{La}$ Paz, Gulf of California, during June 1998. Journal of Marine Systems 30: 305-315.

Obeso-Nieblas M. 2003. Variabilidad espacio-temporal de las condiciones oceanográficas de la Bahía de La Paz, BCS, México. Tesis de Doctorado, Centro Interdisciplinario de Ciencias Marinas, IPN, La Paz, 337 pp.

Obeso-Nieblas M \& AR Jiménez-Illescas. 1989. Propagación de la constituyente $\mathrm{M}_{2}$ de la marea en La Bahía de La Paz, Baja California Sur, México, mediante un modelo bidimensional hidrodinámico numérico. Investigaciones Marinas CICIMAR 4: 241-256.

Obeso-Nieblas M, JH Gaviño-Rodríguez, AR JiménezIllescas \& B Shirasago-Germán. 2002. Simulación numérica de la circulación por marea y viento del noroeste y sur en la Bahía de La Paz, BCS. Oceánides 17: 1-12.

Obeso-Nieblas M, B Shirasago, L Sánchez-Velasco \& JH Gaviño-Rodríguez. 2004. Hydrographic variability in Bahia de La Paz, BCS, Mexico, during the 1997-1998 El Niño. Deep-Sea Research II 51: 689-710.

Obeso-Nieblas M, B Shirasago-Germán, JH GaviñoRodríguez, E Pérez-Lezama, H Obeso-Huerta \& AR Jiménez-Illescas. 2008. Variabilidad hidrográfica en Bahía de La Paz, Golfo de California, México (1995-2005). Revista de Biología Marina y Oceanografía 43(3): 559-567.

Plata L \& A Filonov. 2007. Marea interna en la parte noroeste de la Bahía de Banderas, México. Ciencias Marinas 33(2): 197-215.
Pond S \& GL Pickard. 1983. Introductory dynamical oceanography, $241 \mathrm{pp}$. Pergamon Press, Oxford.

Reiss CS, G Panteleev, CT Taggart, J Sheng \& B de Young. 2000. Observation on larval fish transport and retention on the Scotian Shelf in relation to geostrophic circulation. Fisheries Oceanography 9(3): 195-213.

Roden GI. 1972. Thermohaline structure and baroclinic flow across the Gulf of California entrance and in the Revilla Gigedo Islands region. Journal of Physical Oceanography 22: $177-183$.

Salinas-González F, O Zaytsev \& M Vyacheslav. 2003. Formación de la estructura termohalina del agua en la Bahía de La Paz de verano a otoño. Ciencias Marinas 29(1): 5165.

Sánchez-Velasco L, E Beier, C Avalos-García \& MF Lavín. 2006. Larval fish assemblages and geostrophic circulation in Bahia de La Paz and the surrounding southwestern region of the Gulf of California. Journal of Plankton Research 28(11): 1081-1098.

Silva N \& A Valdenegro. 2003. Evolución de un evento de surgencia frente a punta Curaumilla, Valparaíso. Investigaciones Marinas 31(2): 73-89.

Simpson JH. 1981. The shelf sea front: Implication of their existence and behavour. Philosophical Transations of the Royal Society of London 302: 531-546.

Somarakis S, P Drakopoulos \& V Filippou. 2002. Distribution and abundance of larval fish in the northern Aegean Sea-eastern Mediterranean in relation to early summer oceanographic conditions. Journal of Plankton Research 24(4): 339-357.

Torres-Orozco E. 1993. Análisis volumétrico de las masas de agua del Golfo de California. Tesis de Maestría, Centro de Investigación Científica y de Educación Superior de Ensenada, Baja California, México, 80 pp.

UNESCO. 1991. Processing of oceanographic station data, 138 pp. JPOTS Edition Panel, Paris.

Recibido el 12 de septiembre de 2013 y aceptado el 24 de junio de 2014

Editor Asociado: Mauricio Landaeta D. 